\title{
From Correlative Microscopy to 3D Understanding of Material Microstructures
}

\author{
Frank Muecklich $^{1,2}$, Dominik Britz ${ }^{2}$ and Michael Engstler ${ }^{1,2}$. \\ 1. Saarland University, Dept. Mat.Sci. \& Eng., Saarbruecken, Germany \\ 2. Materials Engineering Center Saarland, Steinbeis Research Center, Saarbruecken, Germany
}

The investigation of the origin and formation of microstructures and the effect that microstructure has on the properties of materials are questions of ever-increasing importance in the strongly growing variety of tailor-made and high performing structural as well as functional materials. The individual ordered regions, the phases and defects, with all their chemical and structural variety, form the complex set of components of the microstructure of a material. The properties of a material are determined not only by the kinds and fractions of components present, but also by details of their geometry; and geometrical analysis can very often prove the key to quantitative understanding the formation of microstructure as well as resulting material properties [1].

In the technological development of materials and routine quality control of microstructures, all of the most important judgments regarding spatial microstructures are still made on the basis of rather traditional two-dimensional microscopic images of metallographic or materialographic cross sections. But increasingly narrow tolerances are being set also on the geometrical appearance of their microstructures requiring not only a considerably more accurate appraisal but an unquestionable and objective quantification with best possible reliability. This requires adequate sensitivity to ensure that even the most subtle effects that slight changes in the manufacturing parameters might have on the spatial microstructure as well as on the properties of the material are detected [2].

In this presentation it will be shown how to get rather comprehensive microstructure information by advanced but classic metallographic preparation accompanied by steps of correlative microscopy with modern scanning analysis techniques. Exploiting the special advantage of considerably large field of view sizes in metallographic techniques and combining that with highest possible resolution in metallographic serial sectioning the implementation of powerful 3D tomography is possible.

Exemplary, this approach is applied on low carbon steel. One of the main challenges in this field is the distinction between the different ferrite forms from proeutectoid to bainitic ferrite [3]. In a first step, contrast formation by etchings was investigated systematically. After that, a correlative study of the complex metallographic effects with Laser Scanning Microscopy as well as Electron Backscatter Diffraction and also of the mechanical properties with statistical nanoindentation has been done (Fig.1). Based on this comprehensive 2D investigation the combination with high resolution metallographic serial sectioning (inspired by [4]) offers the way to 3D understanding of this kind of complex microstructure (Fig.2).

For the given example the color etching procedure is ablation-free and secures best possible depth resolution of serial sectioning and also 3D imaging. The 2D color etching itself gives information about grain orientation in a large field of view, which was initially verified by EBSD. Some kind of grain orientation information is also essential to reconstruct a reliable 3D microstructure. The nanoindentation pattern gives rise to modeling of mechanical properties of the multiphase $3 \mathrm{D}$ grain ensemble. 


\section{References:}

[1] J Ohser, F Muecklich, "Statistical Analysis of Microstructures in Materials Science”, John Wiley \& Sons, Chichester, UK, 2000

[2] F Muecklich, M Engstler, D Britz, J Barrirero, P Rossi, Pract. Metallogr. 52(2015)9, p. 507-524

[3] HK Bhadesia, R Honeycombe, "Steels, Microstrucuture and Properties", ( $3{ }^{\text {rd }}$ edition) ButterworthHeinemann, Cambridge, UK, 2006

[4] J Alkemper, PW Voorhees, Journal of Microscopy 201(2001)3, 388-394

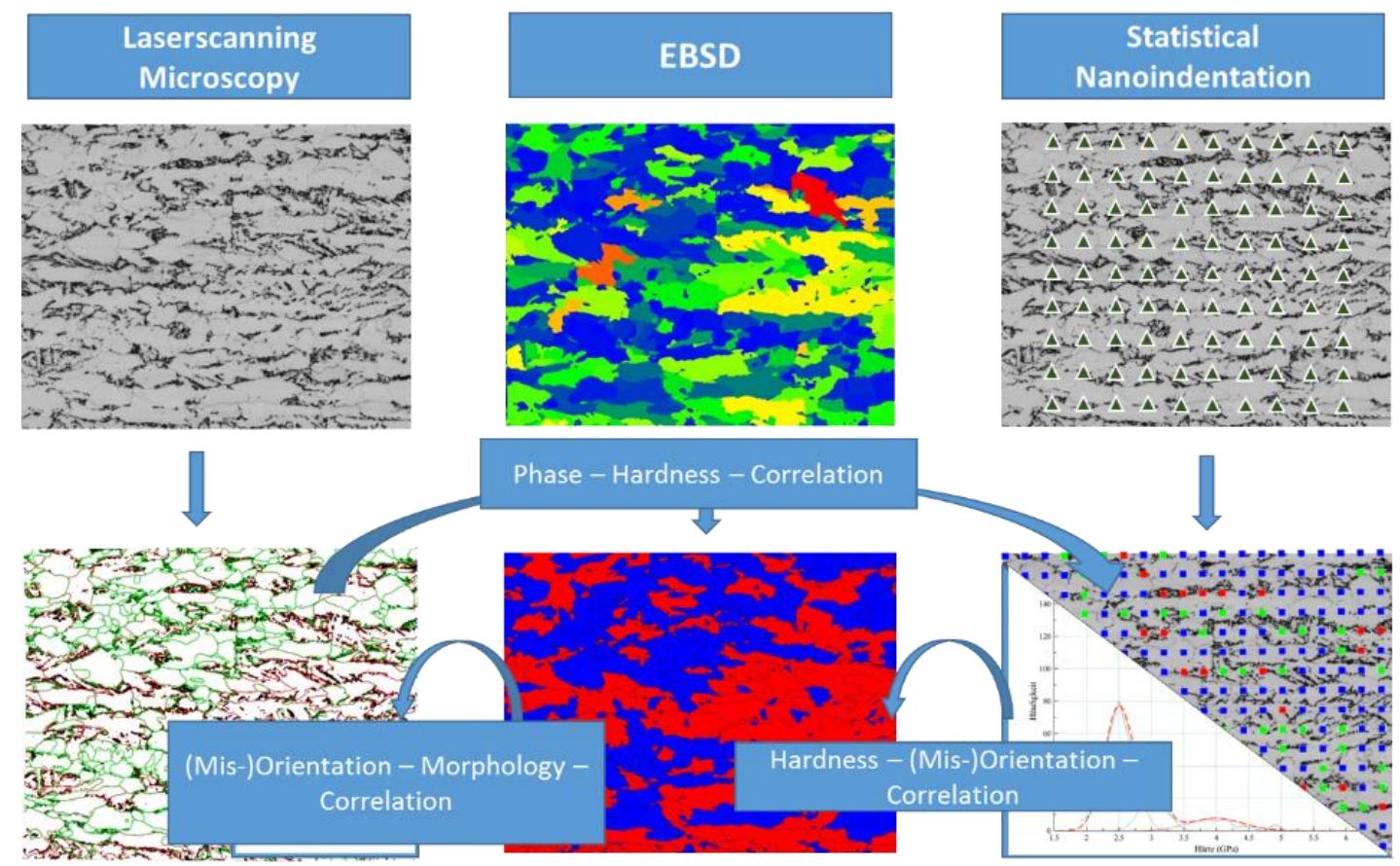

Figure 1. Correlation of morphology (by Laserscanning Microscopy), misorientation (by EBSD) and hardness (by Nanoindentation) to find both phase- and microstructural correlation for a reliable and holistic 3D characterization.
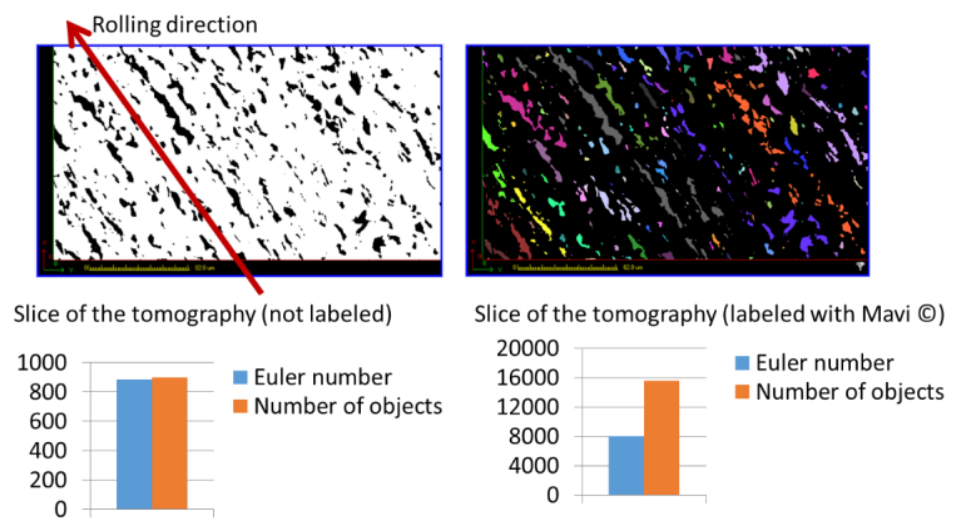

Figure 2. The developed technique could reach a high voxel resolution due to high depth resolution in slicing $(0.42 \pm 0.038 \mu \mathrm{m})$ as well as lateral contrasting and imaging in large field of view size. 3D Euler number is a proof of long-range connectivity in 3D which quantifies the complex spatial morphology with strong implications for texture and mechanical properties as a consequence of sophisticated process control. 